\begin{tabular}{|c|c|}
\hline Title & $\begin{array}{l}\text { Electromagnetic, Thermal, and Mechanical Quench Simulation of NI REBCO Pancake Coils for High Magnetic Field } \\
\text { Generation }\end{array}$ \\
\hline Author(s) & Noguchi, So \\
\hline Citation & $\begin{array}{l}\text { IEEE transactions on applied superconductivity, 29(5), } 4602607 \\
\text { https://doi.org/10.1109/T A SC.2019.2904317 }\end{array}$ \\
\hline Issue Date & $2019-08$ \\
\hline Doc URL & http:/hdl.handle.net/2115/74530 \\
\hline Rights & $\begin{array}{l}\text { (c) } 2019 \text { IEEE. Personal use of this material is permitted. Permission from IEEE must be obtained for all other uses, in } \\
\text { any current or future media, including reprinting/republishing this material for advertising or promotional purposes, } \\
\text { creating new collective works, for resale or redistribution to servers or lists, or reuse of any copyrighted component of } \\
\text { this work in other works. }\end{array}$ \\
\hline Tyре & article (author version) \\
\hline File Information & Final Version_89283.pdf \\
\hline
\end{tabular}

Instructions for use 


\title{
Electromagnetic, Thermal, and Mechanical Quench Simulation of NI REBCO Pancake Coils for High Magnetic Field Generation
}

\author{
So Noguchi
}

\begin{abstract}
The no-insulation (NI) winding technique drastically enhances the thermal stability of $\mathbf{R E B a}_{2} \mathrm{Cu}_{3} \mathbf{0}_{y}$ (REBCO) coils. Even if a larger current than a critical current carried in NI REBCO pancake coils, the coils would not burn out. So far, many NI REBCO pancake coils showed a "self-protecting" characteristic in experiments. However, most of these experiments have been done under a low magnetic field or cooled by liquid nitrogen. Under these conditions, a huge electromagnetic force does not occur after an NI REBCO pancake coil transitions into a normal state.

Recently, the NI winding technique has been applied to high field magnets, such as NMR and accelerators. Under a high magnetic field, a huge hoop stress are generated after one NI REBCO pancake coil constituting a high field magnet by stacking reaches to quench. The huge hoop stress causes the delamination of REBCO coated conductors. That is, the quench protection condition of high field NI REBCO magnets is decided according to the mechanical condition, even though the magnets show a high thermal stability. In this paper, 3 simulation models for NI REBCO pancake coils are compared, and then the mechanical condition is discussed for a quench protection through an electromagnetic, thermal, and mechanical simulation of multistacked NI REBCO pancake coils for high field generation.
\end{abstract}

Index Terms-Equivalent electric circuit model, REBCO magnet, screening current, simulation.

\section{INTRODUCTION}

$\mathbf{T}$ $\mathrm{HE}$ second generation high-temperature superconducting $\left(\mathrm{REBCO} ;(\mathrm{RE}) \mathrm{Ba}_{2} \mathrm{Cu}_{3} \mathrm{O}_{y}(\mathrm{RE}=\right.$ Rare Earth$\left.)\right)$ magnets have a high potential to generate a high magnetic field. In 2018, the small REBCO magnet (named "LBC3") generated 14.5 $\mathrm{T}$ inside of $31 \mathrm{~T}$ field at National High Magnetic Field Lab., USA. A field of $31.5 \mathrm{~T}$ is a world record of $\mathrm{DC}$ magnetic field [1]. MIT, RIKEN, and other institutes have been developing NMR REBCO magnets generating a magentic field higher than $30 \mathrm{~T}$ [2].

The no-insulation (NI) winding technique [3] greatly contributes a high magnetic field generation. The NI technique drastically improves the thermal stability of REBCO magnets. Even if the NI REBCO magnets quenched or a current exceeded the coil critical current, the NI REBCO magnets were

Manuscript receipt and acceptance dates will be inserted here. This work was supported by JSPS KAKENHI under Grant 15KK0192. (Corresponding author: So Noguchi.)

S. Noguchi is with Graduate School of Information Science and Technology, Hokkaido University, Sapporo 060-0814, Japan (e-mail: noguchi@ssi.ist.hokudai.ac.jp).

Color versions of one or more of the figures in this paper are available online at http://ieeexplore.ieee.org.

Digital Object Identifier will be inserted here upon acceptance. protected from burning-out, as a function of "self-protecting." The NI technique made the way to generate a high magnetic field with REBCO magnet.

With the development of the NI winding technique, simulation methods for NI REBCO magnet have also been developed. The first simulation model consists of a parallel circuit of the coil inductance and the turn-to-turn contact resistance [3]. Next, some complicated models were proposed such as the distributed network model [4], the partial element equivalent circuit (PEEC) model [5], the distributed resistance model [6], and so on. In these models, a pancake coil is azimuthally subdivided to some elements which are represented by local inductance and contact resistance. These model are basically the same, and the derivation method of local inductances is different; however the inductance components are neglected in [6]. In addition, some complicated methods were extended to take into account the thermal and stress phenomena [5], [7], [8]. With the development of the simulation technique, the high thermal stability of REBCO pancake coils were proven, because the current flows in the coil-radial direction preventing form the local normal zone. However, recently, a problem on mechanical stability arised for high magnetic field generation.

First of all, the features of the simulation methods are introduced, compared, and discussed in this paper. And then, the stress weakness after quench is shown, and the condition of quench protection is also discussed.

\section{NI REBCO Magnet Simulations}

There are 3 types of no-insulation (NI) REBCO magnet simulation models; the simple, the moderate, and the complicated models, as shown in Fig. 1. The features of these models are explained below.

\section{A. Simple model}

The simple model consists of a radial component of turnto-turn contact resistance and a azimuthal component of a coil inductance and a REBCO tape resistance for one pancake coil [3]. The equation to be solved is as follows:

$$
L \frac{\mathrm{d} I_{\theta}}{\mathrm{d} t}+\frac{R_{\mathrm{sc}} R_{\mathrm{mt}}}{R_{\mathrm{sc}}+R_{\mathrm{mt}}} I_{\theta}=R_{\mathrm{ct}}\left(I_{\mathrm{op}}-I_{\theta}\right)
$$

where $L, I_{\theta}, R_{\mathrm{sc}}, R_{\mathrm{mt}}, R_{\mathrm{ct}}$, and $I_{\mathrm{op}}$ are the coil inductance, the azimuthal current, the REBCO layer resistance, the matrix resistance, the contact resistance, and the operating current, respectively. The coil inductance $L$ is easily computed by a 


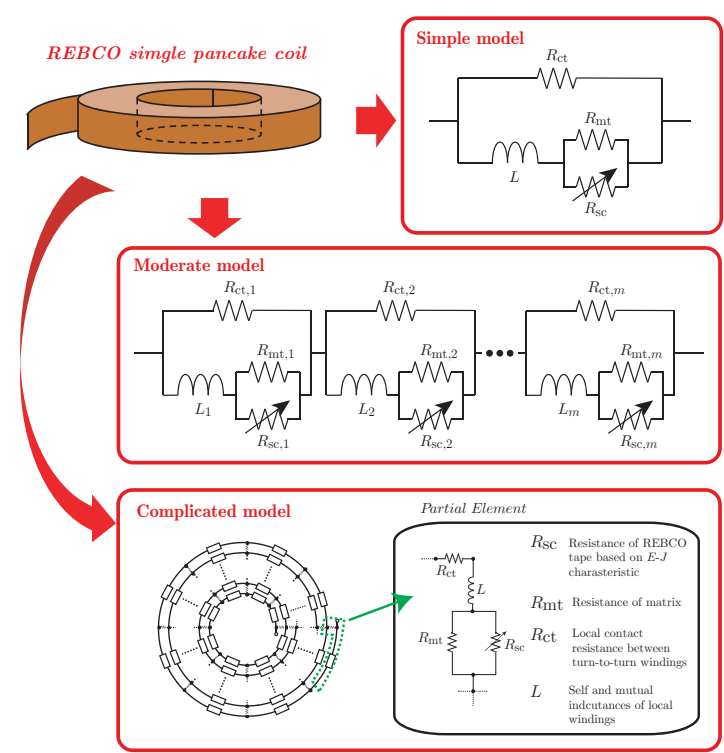

Fig. 1. NI REBCO simulation models; the simple, the moderate, and the complicated.

method in [9], and the turn-to-turn contact resistance $R_{\mathrm{ct}}$ is obtained by measurement or calculated by

$$
R_{\mathrm{ct}}=\sum_{i=1}^{N-1} \frac{\rho_{\mathrm{ct}}}{2 \pi r_{i} w}
$$

where $N, \rho_{\mathrm{ct}}, r$, and $w$ are the number of turns, the turnto-turn contact resistivity, radius, and REBCO tape width, respectively [10].

To couple with the thermal simulation, since the heat conduction cannot be taken into account, the following governing equation is used:

$$
\rho c \frac{\partial T}{\partial t}=q
$$

where $\rho, c, T$, and $Q$ are the mass density, the specific heat, the temperature, the heating per volume $\left(\int q \mathrm{~d} v=\frac{R_{\mathrm{sc}}+R_{\mathrm{tm}}}{R_{\mathrm{sc}} R_{\mathrm{mt}}} I_{\theta}^{2}+\right.$ $\left.R_{\mathrm{ct}}\left(I_{\mathrm{op}}-I_{\theta}\right)^{2}\right)$, respectively. The temperature is uniform in each pancake coil. Here, the cooling condition is ignored, that is, an adiabatic condition is taken.

To compute the stress and strain in the coil, the analytical solution is given in [11] when it is assumed that the magnetic field inside a pancake coil linearly changes. The governing equation to be solved is

$$
r \frac{\partial \sigma_{r}}{\partial r}+\sigma_{r}-\sigma_{\theta}+r J_{\theta} B_{z}(r)=0
$$

where $r, \sigma_{r}, \sigma_{\theta}, J_{\theta}$, and $B_{z}$ are the radial position, the radial and hoop stresses, the azimuthal current, and $z$ component of magnetic field, respectively. The strains, $\epsilon_{r}, \epsilon_{\theta}, \epsilon_{z}$ in the $r, \theta$, and $z$ directions, are given by

$$
\begin{aligned}
\epsilon_{r} & =\frac{\sigma_{r}}{E_{r}}-\nu_{\theta r} \frac{\sigma_{\theta}}{E_{\theta}}-\nu_{z r} \frac{\sigma_{z}}{E_{z}} \\
\epsilon_{\theta} & =\frac{\sigma_{\theta}}{E_{\theta}}-\nu_{r \theta} \frac{\sigma_{r}}{E_{r}}-\nu_{z \theta} \frac{\sigma_{z}}{E_{z}} \\
\epsilon_{z} & =\frac{\sigma_{z}}{E_{z}}-\nu_{r z} \frac{\sigma_{r}}{E_{r}}-\nu_{\theta z} \frac{\sigma_{\theta}}{E_{\theta}}
\end{aligned}
$$

where $E_{r}, E_{\theta}$, and $E_{z}$ are the Young's moduli, respectively, in the $r, \theta$, and $z$ directions, and $\nu_{12} \equiv-\epsilon_{2} / \epsilon_{1}$ is the Poisson's ratio, respectively. The strain linearly changes in the radial direction.

As a boundary condition, $\sigma_{r}=0$ is given at the inner and outer surfaces of each pancake coil.

\section{B. Moderate model}

In the moderate models, one pancake coil is radially subdivided, and one element represents 1 - a few tens turns, as shown in Fig. 1. The system equations for the equivalent circuits is as follows:

$$
\sum_{j=1}^{m} L_{i, j} \frac{\mathrm{d} i_{\theta, j}}{\mathrm{~d} t}+\frac{R_{\mathrm{sc}, i} R_{\mathrm{mt}, i}}{R_{\mathrm{sc}, i}+R_{\mathrm{mt}, i}} I_{\theta, i}=R_{\mathrm{ct}, i}\left(I_{\mathrm{op}}-I_{\theta, i}\right)
$$

where $m$ is the number of elements, and

$$
R_{\mathrm{ct}, i}=\left(\frac{N}{m}-\beta_{i}\right) \frac{R_{\mathrm{ct}}^{\exp }}{N-1}=\sum_{j=i}^{i+N / m} \gamma_{j} \frac{\rho_{\mathrm{ct}}}{2 \pi r_{j} w}
$$

where $R_{\mathrm{ct}}^{\exp }$ is the measured contact resistance, and

$$
\begin{gathered}
\beta_{i}=\left\{\begin{array}{cl}
0.5 & i=1, m \\
0 & \text { otherwise }
\end{array}\right. \\
\gamma_{j}=\left\{\begin{array}{cl}
0.5 & j=1, m \\
1 & \text { otherwise }
\end{array} .\right.
\end{gathered}
$$

The thermal equation to be solved is represented by

$$
2 \pi w \rho c \int \frac{\partial T}{\partial t} r \mathrm{~d} r=2 \pi w \lambda \int \frac{\partial^{2} T}{\partial r^{2}} r \mathrm{~d} r+Q
$$

where $\lambda$ is the thermal conductivity. (10) is solved with the finite element method (FEM). It is supposed to be adiabatic in (10).

In order to obtain the stress, (4) is also solved with FEM, and the strains are derived from (5). The temperature and the strain linearly changes in each finite element.

\section{Complicated model}

The complicated model represents one pancake coil with many azimuthally subdivided elements along with REBCO tape. Each element consists of a local inductance and a REBCO tape resistance, and the elements are radially connected with the contact resistance, as shown in Fig. 1. The equivalent circuit model is computed by the following equations:

$$
\begin{aligned}
& I_{\mathrm{L}, i}-I_{\mathrm{L}, i-1}+I_{\mathrm{ct}, i}-I_{\mathrm{ct}, i-d}=0 \quad\left(I_{0}=I_{m+1}=I_{\mathrm{op}}\right) \\
& \sum_{j=i}^{i-d-1}\left(\sum_{k=1}^{n} L_{j, k} \frac{\mathrm{d} I_{L, k}}{\mathrm{~d} t}+\frac{R_{\mathrm{sc}, j} R_{\mathrm{mt}, j}}{R_{\mathrm{sc}, j}+R_{\mathrm{mt}, j}}\right)=I_{\mathrm{ct}, i} R_{\mathrm{ct}, i}
\end{aligned}
$$

where $i, d$, and $n$ are, respectively, the element number, the number of circumferential division, and the number of elements, and

$$
R_{\mathrm{ct}, i}=\frac{R_{\mathrm{ct}}^{\exp }}{d(N-1)}=d \frac{\rho_{\mathrm{ct}}}{2 \pi r w} .
$$


TABLE I

Comparison of Simple, Moderate, and Complicated Models of NI REBCO PANCAKE COILS

\begin{tabular}{|c|c|c|c|}
\hline & Simple & Moderate & Complicated \\
\hline Accuracy & $x$ & o & () \\
\hline Computation time & (?) & $\circ$ & $x$ \\
\hline Memory usage & (?) & ○ & $x$ \\
\hline Inductance computation & () & $\triangle$ & $x$ \\
\hline Local hotspot & $x$ & ○ & () \\
\hline SCIF computation & ○ & 0 & $\times$ \\
\hline Thermal computation & $\triangle$ & ○ & ० \\
\hline Stress \& strain computation & $\circ$ & ○ & $\circ$ \\
\hline
\end{tabular}

The thermal and mechanical phenomena are simulated with FEM for the following equation and (4), separately.

$w \rho c \iint \frac{\partial T}{\partial t} r \mathrm{~d} r \mathrm{~d} \theta=w \iint\left(\lambda_{r} \frac{\partial^{2} T}{\partial r^{2}}+\lambda_{\theta} \frac{\partial^{2} T}{\partial \theta^{2}}\right) r \mathrm{~d} r \mathrm{~d} \theta+Q$

where $\lambda_{r}$ and $\lambda_{\theta}$ are the thermal conductivity in the radial and circumferential direction, respectively. The temperature and the strain linearly changes in each finite element .

\section{Comparison between models}

As mentioned above, the thermal, mechanical, and electromagnetic phenomena can be obtained using each model. However, a local hotspot cannot be represented by only the simple model. The screening current-induced field (SCIF) computation is proposed for the simple and the moderate methods [12], but there is no method to compute the SCIF with the complicated method so far. Because, a circular current is assumed in the SCIF computation method in proposed [12], therefore the proposed method cannot apply to the complicated method where the tapes are azimuthally subdivided.

Table I shows the comparison of the features of the simple, the moderate, and the complicated models. The moderate models are a totally balanced method.

\section{Simple, Moderate, And Complicated Model SIMULATIONS}

Table II lists the specifications of the simulated NI REBCO magnet, which consists of 6 double pancake (DP) coils in series connection, as shown in Fig. 2. The magnet is supposed to be operated under an external field of $20 \mathrm{~T}$. As a quench simulation, the case that the bottom single pancake coil (SP \#12) entirely quenches at $t=0$ is simulated with the simple, the moderate, and complicated models (i.e. the REBCO resistance in the normal state is set on the quenched pancake coil in this simulation). Although a local normal zone is likely to appear, in order to evaluate a worst quench scenario, the quench of whole SP \#12, whose critical current is the lowest among pancake coils, is supposed, and a reason to quench is not specified in this paper. For the moderate models, the SPs are radially divided into 10 or 20 , i.e. 10 or 20 parallel equivalent circuits express one single pancake (SP) coil, called the moderate-10 and moderate- 20 models, respectively. The division numbers of each model are shown in Table III.

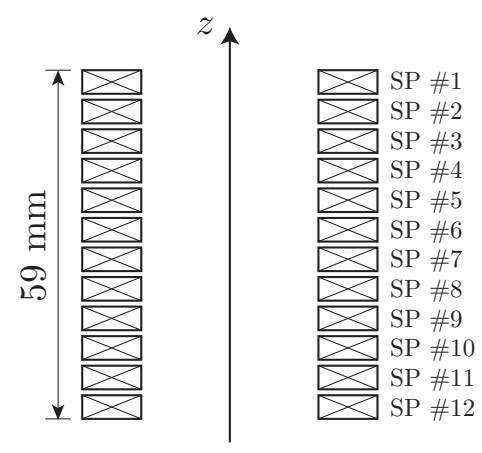

Fig. 2. Simulation model of NI REBCO magnet consisting of 6 double pancake coils.

TABLE II

SPECIFICATIONS OF NI REBCO COIL AND REBCO TAPE

\begin{tabular}{lc}
\hline Number of DPs & 6 \\
Coil i.d.: o.d & $60 ; 100$ \\
Coil height (tape width) $(\mathrm{mm})$ & 4.0 \\
Number of turns & 200 \\
Contact resistivity $\left(\mu \Omega \cdot \mathrm{cm}^{2}\right)$ & 70 \\
Operating current $(\mathrm{A})$ & 200 \\
Magnet constant $(\mathrm{mT} / \mathrm{A})$ & 30.4 \\
Temperature $(\mathrm{K})$ & 4.2 \\
External field $(\mathrm{T})$ & 20 \\
\hline \hline Tape width $(\mathrm{mm})$ & 4.0 \\
Tape thickness $(\mathrm{mm})$ & 0.1 \\
REBCO layer thickness $(\mu \mathrm{m})$ & 1.0 \\
Copper matrix thickness $(\mu \mathrm{m})$ & 20 (both sides) \\
Critical current at $77 \mathrm{~K}$, s.f. $(\mathrm{A})$ & 115 \\
\hline
\end{tabular}

As a cooling condition, an adiabatic condition is assumed at $4.2 \mathrm{~K}$. The anisotropic thermal conductivity and the specific heat of the pancake coils are temperature-dependent in the simulation. The thermal contact resistance between REBCO tapes are considered, however the other ones (such as between REBCO layer and matrix) are not. It is supposed to be thermally insulated between pancake coils. The Young's moduli $\left(E_{r}, E_{\theta}\right.$, and $\left.E_{z}\right)$ in the radial, circumferential, and axial directions are 69,144 , and $144 \mathrm{GPa}$, respectively. The axial mechanical boundary condition is not considered in the simulation. Any condition given by a power supply is not considered, too.

\section{A. Azimuthal current}

Fig. 3 shows the average azimuthal currents in each SP coil with the on-axis fields by the simple, the moderate-10, the moderate-20, and the complicated models. The results of the moderate-10, -20 , and complicated models represents the average azimuthal currents in each SP coil. The profiles of the simple and moderate-10 models looks very similar, and the quench fast propagates from SP \#10 to \#1 within $0.15 \mathrm{~s}$,

TABLE III

Division Numbers of ONE Single PANCAKE

\begin{tabular}{lcccc}
\hline Direction & Simple & Moderate-10 & Moderate-20 & Complicated \\
\hline Radial & 1 & 10 & 20 & 200 \\
Azimuthal & - & - & - & 4 \\
\hline
\end{tabular}




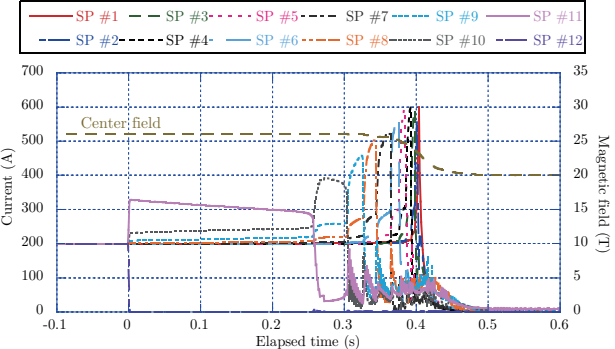

(a)

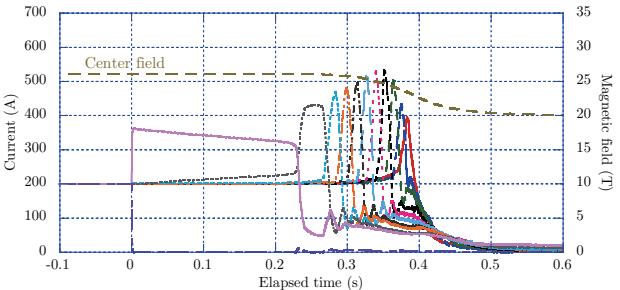

(b)

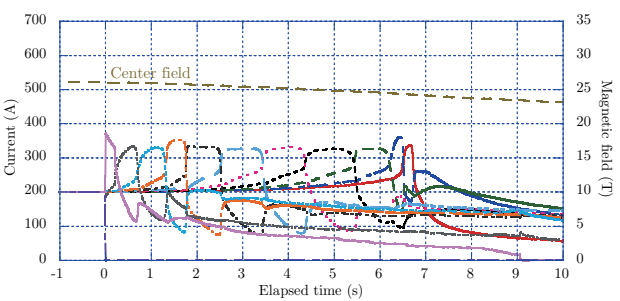

(c)

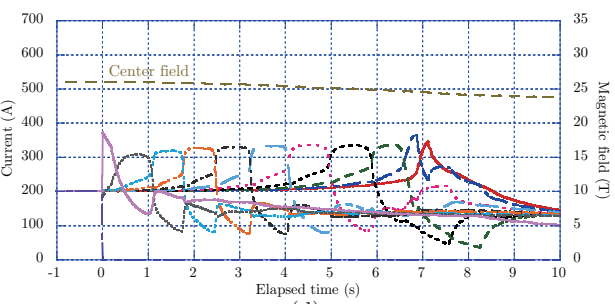

(d)

Fig. 3. Simulation results of average azimuthal current in each SP coil and onaxis field; (a) simple model, (b) average current in moderate-10, (c) moderate20, and (d) complicated models.

mainly due to the induced current. With the proceeding of the continuous quenches, the induced currents increase. Hence, the quench propagation velocity is faster on the late quench occurrence. The on-axis field begins to decrease at the quench of SP \#6.

Meanwhile, the velocity of the quench propagation is very slow in the case of the moderate-20 and complicated models. The induced current in each coil does not increase with quench propagation, and the azimuthal currents of \#1 - \#11 reach to approximately $350 \mathrm{~A}$. The on-axis field gradually decreases during the quench propagation.

The velocity difference of Fig. 3 (b) and (c) is caused by the magnet coupling between elements in each models. The detail explanation is shown in the following sections.

\section{B. Temperature}

Fig. 4 shows the maximum temperature on each SP coil with time, simulated with the moderate-10 and complicated models. In the moderate-10 model simulation, the temperature

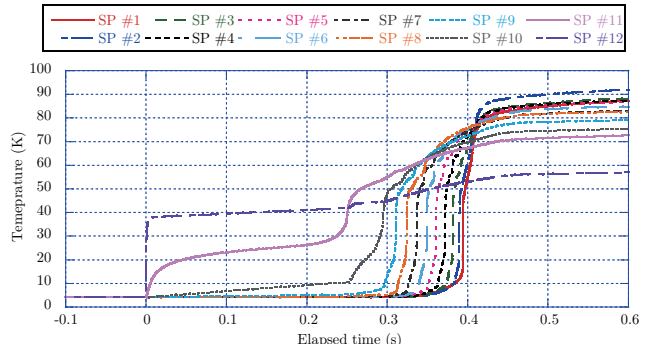

(a)

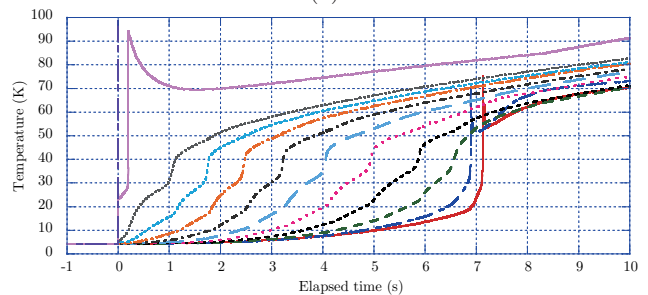

(b)

Fig. 4. Maximum temperature transition of each SP coil; (a) moderate-10 and (b) complicated model. (a)

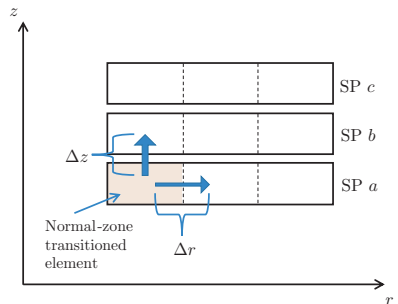

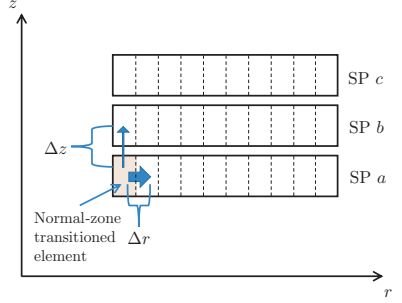

(b)
Fig. 5. Quench propagation direction depending on element size. An induced current propagates toward an element with a strong magnetic coupling.

of \#1 - \#10 increases rapidly. In the complicated model, we can observe the slow increase in temperature. The difference of the quench propagation is caused by the division size. Since the velocity of the induced-current propagation due to magnetic coupling is much faster than the temperature diffusion, the quench propagation is cased by the inducedcurrent propagation.

Fig. 5 shows the mechanism of the induced-current propagation in the moderate-10 and -20 models. When the division is rough (a small number of divisions), the normal-zone transitioned element is closer to the element in the adjacent SP coils than that of the adjacent element in the same SP coil. As shown in Fig. 5(a), when $\Delta z<\Delta r$, since the magnetic coupling between the innermost elements of SPs $a$ and $b$ is strong, a large current is induced in the innermost element in SP $b$ in the simulation. That is, the induced current (quench) faster propagates in the vertical direction than in the radial direction.

On the other hand, when $\Delta z>\Delta r$, the magnetic coupling in the radial direction is stronger. Therefore, the induced current largely propagates in the radial direction. Fig. 6 represents the current distribution transition on each turns of SP \#11 just after the SP \#12 quench. The large currents are induced on the innermost and outermost turns due to the inductance distribution, and then the currents diffuse toward the coil-mid 


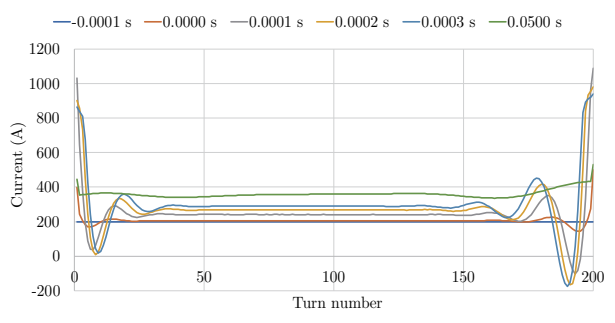

Fig. 6. Azimuthal current on each turn of SP \#11 after SP \#12 quench. At $t=0.0001 \mathrm{~s}$, a large current is induced at the innermost and outermost turns. And then, the current diffuses toward the coil-mid turns. At $t=0.05 \mathrm{~s}$, the current diffusion is almost accomplished.

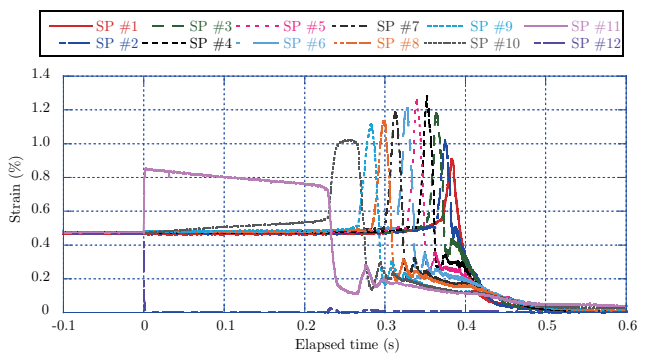

(a)

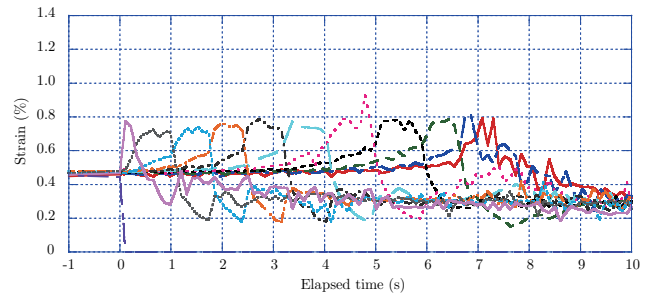

(b)

Fig. 7. Simulation result of maximum strain on each SP coil; (a) moderate-10 and (b) complicated models.

turns. The induced current makes a Hoop stress stronger, and the temperature rises up there due to the radial current. Since the simple model cannot represent such a current diffusion, the quench propagation velocity is overestimated.

\section{Mechanical stress and strain}

As explained above, the large currents in the circumferential direction are induced in the SP coils next to a quenched SP coil. It is well known that a large current in a strong magnetic field deteriorates the critical current of SP coil, due to peeling the REBCO layer from the Hastelloy substrate [13]. In general, the threshold of REBCO layer peeling is $0.4-0.5 \%$ in strain.

Fig. 7 shows the strain transitions in the moderate-10 and complicated models. Since the 6 NI REBCO DP coils works as an insert magnet, an external magnetic field of $20 \mathrm{~T}$ is applied during the sequential quenches. As a result, a large strain is generated in all the SP coils except for the initially quenched coil (SP \#12). That is, the REBCO tapes of the SP coils \#1 - \#11 are irreversibly mechanically damaged when the strain threshold is supposed to be $0.5 \%$ in this paper.

Indeed, when the strain exceeds the threshold, the coil critical current $I_{\mathrm{c}}$ would be immediately reduced. However,

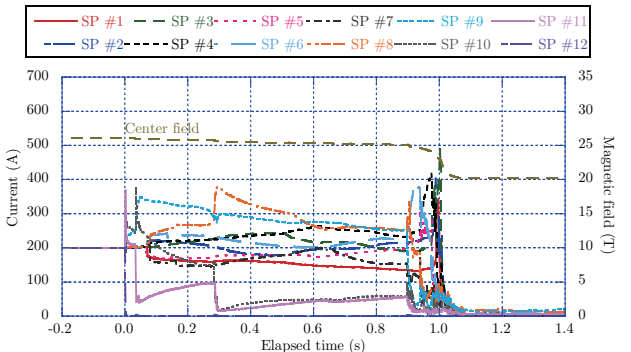

(a)

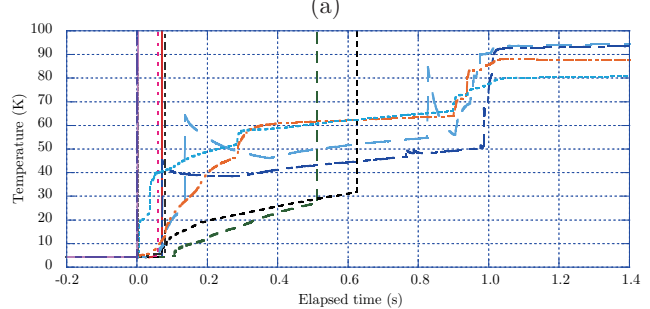

(b)

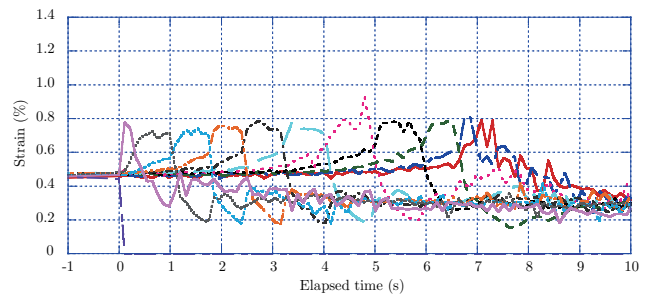

(c)

Fig. 8. Simulation results in case of critical-current degradation due to large strain; (a) average azimuthal current, (b) temperature, and (c) strain. When the strain of element exceeds $0.5 \%$, the critical current decreases to $30 \%$ there.

since the $I_{\mathrm{c}}$ degradation mechanism is not clear, the damage by large strain cannot correctly be taken into account in this paper. Here, it is assumed that the critical current $I_{\mathrm{c}}$ decreases to $30 \%$ after the strain exceeds $0.5 \%$. The roughly $30 \%$ degradation in the critical current of the insert REBCO magnet generating 45.5 $\mathrm{T}$ was measured with the YateStar after unwinding [1]. Fig. 8 shows the simulation results considering the $30 \% I_{\mathrm{c}}$ degradation at elements where the strain exceeds $0.5 \%$. As we can observe, the quench fast propagates from SP to SP. The $I_{\mathrm{C}}$ degradation due to mechanical damage makes the quench propagation accelerate. The temperature rises up higher than the simulation results of Fig. 4. From Fig. 8(c), the strain largerer than $0.5 \%$ can be observed.

For accurate simulation, the phenomenon of $I_{\mathrm{c}}$ degradation must be investigated, and then the mechanical degradation model will be established. In addition, when a large stress is generated, we have to reocnsider the stress simulation model, such as a boundary condition.

\section{Protection from Large Strain}

In the world record DC magnet (the $\mathrm{LBC} 3$ insert), the quench propagated from the bottom to the top pancake coils within a very short time [8], as well as the irreversible critical-current degradation happened at the same time [1]. As mentioned above, the mechanical $I_{\mathrm{c}}$ degradation accelerates the quench propagation. Although the NI winding technique drastically improves the thermal stability of REBCO magnet, it 


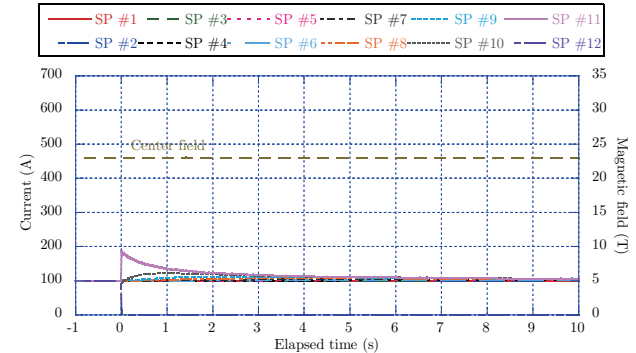

(a)

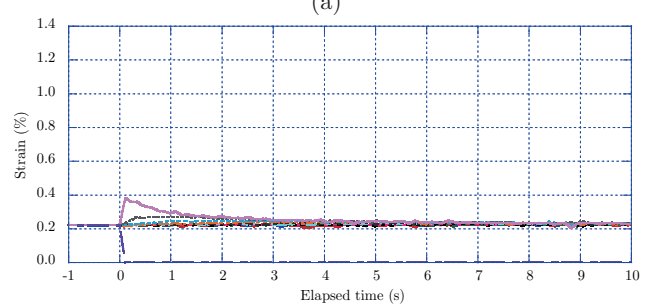

(b)

Fig. 9. Simulation result of azimuthal current of each SP coil and on-axis field; (a) simple model, (b) average current in moderate model, and (c) average current in complicated model.

is necessary to protect the REBCO magnet from $I_{\mathrm{c}}$ degradation due to large strain in order to generate a high magnetic field.

Commonly, the operating current is constrained according to the critical current when operating at relatively high temperature or in relatively low magnetic field. For high magnetic field generation at extremely low temperature, the operating current is limited due to the strain. However, an extra current induced in NI magnet during quench damages the REBCO tape.

Therefore, it is necessary to decide the operating current not to exceeds the strain limitation by adding the induced current. It is seen from Figs. 3(c), (d), and 8 that the amount of induced current is almost the same as the operating current. Hence, when the strain limitation is defined as $\epsilon_{\theta}^{\text {limit }}$ at current $I^{\text {limit; }}$

$$
\epsilon_{\theta}^{\text {limit }}=\epsilon_{\theta}\left(I^{\text {limit }}\right),
$$

the conservative operating current $I_{\mathrm{op}}$ must be the half of $I^{\text {limit }}$

$$
I_{\mathrm{op}}=\frac{1}{2} I^{\text {limit }}
$$

Fig. 9 presents the simulation result in the case of the half operating current $\left(I_{\mathrm{op}}=100 \mathrm{~A}\right)$. The simulation condition is the same as Chapter III, except for the operating current. After SP \#12 quench, the net azimuthal current (operating + induced current) in SP \#11 is lower than the operating current limitation $I^{\text {limit }}$ given by the strain limitation $\epsilon_{\theta}^{\text {limit }}$. Surely, the strain of SP \#11 does not exceed the limitation.

\section{CONCLUSION}

In this paper, 3 simulation methods for quench simulation of multistacked no-insulation (NI) REBCO single pancake (SP) coils are compared; the simple, the moderate, and the complicated models. As the results, since an induced current propagates due to a strong magnetic coupling, the moderate model with sufficient number of elements or the complicated method must be used. The distance between elements in radial and vertical direction is important to represent an accurate quench propagation.

Since a large amount of current is induced in NI REBCO SP coil next to a quenched SP coil, a mechanical damage (peeling REBCO layer from Hastelloy substrate) is a serious problem for high magnetic field generation. The critical-current degradation due to peeling accelerates a quench propagation between SP coils. When the LBC3 insert quenched, the fast quench propagation was observed, in addition with the criticalcurrent degradation [1], [8]. To protect an NI REBCO magnet, the operating current must be the half of the maximum current given by the strain limitation.

To improve the accuracy of quench simulation of NI RE$\mathrm{BCO}$ magnets, it is necessary to clarify the mechanism of the critical-current degradation due to a large strain in near future. Especially, the stress concentration due to screening current must be shown. Therefore, since the accurate screening current distribution is required, I need to improve the SCIF simulation method [12].

\section{APPENDIX A}

\section{COMPUTATION OF ELEMENT INDUCTANCE}

For the moderate and complicated models, it is difficult to obtain the self and mutual inductance of elements. Although the FEM computation is useful, I introduce a numerical computation method.

To compute the self-inductance $L$, the following equations are computed with Gauss-Legendre quadrature;

$$
\begin{aligned}
& L=\frac{1}{I^{2}} \int \boldsymbol{A}(\boldsymbol{x}) \cdot \boldsymbol{J}(\boldsymbol{x}) \mathrm{d} v \\
& \boldsymbol{A}(\boldsymbol{x})=\frac{\mu_{0}}{4 \pi} \int \frac{\boldsymbol{J}\left(\boldsymbol{x}^{\prime}\right)}{\left|\boldsymbol{x}-\boldsymbol{x}^{\prime}\right|} \mathrm{d} v^{\prime}
\end{aligned}
$$

where $I, J, \mu_{0}, \boldsymbol{x}$ and $\boldsymbol{x}^{\prime}$ are the current, the current density, the permeability of free space, the position vectors in the coil volume $v$ and $v^{\prime}$, respectively. It is necessary to use the different number of Gauss nodes between (17) and (18) to avoid zero division in Gauss-Legendre quadrature.

To obtain the mutual inductance $M_{12}$ between elements 1 and 2 , the following equations are also computed with GaussLegendre quadrature;

$$
\begin{gathered}
M_{12}=\frac{E_{m}-\frac{1}{2} L_{1} I_{1}^{2}-\frac{1}{2} L_{2} I_{2}^{2}}{I_{1} I_{2}} \\
E_{m}=\frac{1}{2} \int \boldsymbol{A}\left(\boldsymbol{x}_{1}\right) \cdot \boldsymbol{J}\left(\boldsymbol{x}_{1}\right) \mathrm{d} v_{1}+\frac{1}{2} \int \boldsymbol{A}\left(\boldsymbol{x}_{2}\right) \cdot \boldsymbol{J}\left(\boldsymbol{x}_{2}\right) \mathrm{d} v_{2} \\
\boldsymbol{A}\left(\boldsymbol{x}_{i}\right)=\frac{\mu_{0}}{4 \pi}\left\{\int \frac{\boldsymbol{J}\left(\boldsymbol{x}^{\prime}{ }_{i}\right)}{\left|\boldsymbol{x}_{i}-\boldsymbol{x}^{\prime}{ }_{i}\right|} \mathrm{d} v_{i}^{\prime}+\int \frac{\boldsymbol{J}\left(\boldsymbol{x}^{\prime}{ }_{j}\right)}{\left|\boldsymbol{x}_{i}-\boldsymbol{x}^{\prime}{ }_{j}\right|} \mathrm{d} v_{j}^{\prime}\right\}
\end{gathered}
$$

where $(i, j)=(1,2)$ or $(2,1)$.

\section{ACKNOWLEDGMENT}

The author would like to thank Y. Iwasa (MIT), S. Hahn (SNU/NHMFL), and A. Ishiyama (Waseda Univ.) for valuable discussions. Also, the author would like to thank D. Larbalestier, T. Painter, I. Dixon, K. Kim, K. Kim, K. Bhattarai (NHMFL), D. Park, P. Michael, J. Lee (MIT), S. Kim, H. Ueda (Okayama Univ.), and R. Miyao (Hokkaido Univ.). 


\section{REFERENCES}

[1] S. Hahn, K. Kim, K. Kim, X. Hu, T. Painter, I. Dixon, S. Kim, K. Bhattarai, S. Noguchi, J. Jaroszynski, and D. Larbalestier, "World record DC magnetic field using an $\mathrm{REBa}_{2} \mathrm{Cu}_{3} \mathrm{O}_{\mathrm{x}}(\mathrm{RE}=\mathrm{Y}, \mathrm{Gd})$ superconducting magnet," presented at Coated Conductors for Applications Workshop 2018.

[2] Y. Iwasa, J. Bascuñán, S. Hahn, J. Voccio, Y. Kim, T. Lècrevisse, J. Song, and K. Kajikawa, "A high-resolution 1.3-GHz/54-mm LTS/HTS NMR magnet," IEEE Trans. Appl. Supercond., vol. 25, no. 3, Jun. 2015, Art. no. 4301205.

[3] S. Hahn, D. K. Park, J. Bascuñàn, and Y. Iwasa, "HTS pancake coils without turn-to-turn insulation," IEEE Trans. Appl. Supercond., vol. 21, no. 3, pp. 1592-1595, Jun. 2011.

[4] W. D. Markiewicz, J. J. Jaroszynski, D. V. Abraimov, R. E. Joyner, and A.Khan, "Quench analysis of pancake wound REBCO coils with low resistance between turns," Supercond. Sci. Technol., vol. 29, no. 2, Dec. 2015, Art. no. 025001.

[5] T. Wang, S. Noguchi, X. Wang, I. Arakawa, K. Minami, K. Monma, A. Ishiyama, S. Hahn, and Y. Iwasa, "Analyses of transient behaviors of no-insulation REBCO pancake coils during sudden discharging and overcurrent," IEEE Trans. Appl. Supercond., vol. 25, no. 3, Jun. 2015, Art. no. 4603409.

[6] Y. Yanagisawa, K. Sato, K. Yanagisawa, H. Nakagome, X. Jin, M. Takahashi, and H. Maeda, "Basic mechanism of self-healing from thermal runaway for uninsulated REBCO pancake coils," Phys. C, Supercond., vol. 499, pp. 40-44, Apr. 2014.

[7] R. Miyao, H. Igarashi, A. Ishiyama, and S. Noguchi, "Thermal and electromagnetic simulation of multistacked no-insulation REBCO pancake coils on normal-state transition by PEEC method" IEEE Trans. Appl. Supercond., vol. 28, no. 3, Apr. 2018, Art. no. 4601405.

[8] K. Bhattarai, K. Kim, K. Kim, S. Noguchi, T. Painter, S. Lee, and S. Hahn, "Quench analysis of stacks of no-insulation REBCO coils demonstrating electromagnetic quench propagation and self-protecting behavior," presented at Int. Conf. on Magnet Technology., 2017.

[9] H. W. Beaty and D. G. Fink, Standard Handbook for Electrical Engineers, McGraw-Hill Book, 1949.

[10] X. Wang, S. Hahn, Y. Kiim, J. Bascuñán, J. Voccio, H. Lee, and Y. Iwasa, "Turn-to-turn contact characteristics for an equivalent circuit model of no-insulation ReBCO pancake coil," Supercond. Sci. Technol., vol. 29, no. 2, Dec. 2015, Art. no. 035012.

[11] Y. Iwasa, Case Studies in Superconducting Magnets, Design and Operational Issues, Springer, 1994.

[12] S. Noguchi, D. Park, S. Hahn, and Y. Iwasa, "A simple screening current simulation method using equivalent circuit model for REBCO pancake coils," presented at Applied Superconductivity Conference 2018.

[13] K. Kajita, S. Iguchi, Y. X. Xu, M. Nawa, M. Hamada, T. Takao, H. Nakagome, S. Matsumoto, G. Nishijima, H. Suematsu, M. Takahashi, and Y. Yanagisawa, "Degradation of a REBCO coil due to cleavage and peeling originating from an electromagnetic force," IEEE Trans. Appl. Supercond., vol. 26, no. 4, Jun. 2018, Art. no. 4301106. 